\title{
Supplementary Materials: On the suitability of the Thorpe-Mason model for Calculating Sublimation of Saltating Snow
}

\author{
Varun Sharma ${ }^{1}$, Francesco Comola $^{1}$, and Michael Lehning ${ }^{1,2}$ \\ ${ }^{1}$ School of Architecture, Civil and Environmental Engineering, Swiss Federal Institute of Technology, Lausanne, Switzerland \\ ${ }^{2}$ WSL Institute for Snow and Avalanche Research SLF, Davos, Switzerland
}

Correspondence: Varun Sharma (varun.sharma@epfl.ch)

\section{Introduction}

The contents of this supplement are intended to describe in detail, the numerical methods used in this study, the physical and empirical parameters and constants employed and additional results that complement those presented in the main portion of the letter.

\section{S1 Large-eddy simulation - equations and numerical setup}

\section{S1.1 Flow equations}

The large-eddy simulation (LES) technique solves three-dimensional filtered Navier-Stokes (N-S) equations in rotational form for an incompressible flow in hydrostatic equilibrium along with the continuity equation. Advection-diffusion equations for temperature and specific humidity are solved and resulting buoyancy forces are added to the N-S equations using the Boussinesq approximation. The equations solved (presented using Einstein's convention) are as follows.

$$
\begin{aligned}
\partial_{t} \tilde{u}_{i}+\widetilde{u}_{j}\left(\partial_{j} \widetilde{u}_{i}-\partial_{i} \widetilde{u}_{j}\right) & =-\frac{1}{\rho_{f}} \partial_{i} \widetilde{p_{\infty}} \delta_{i, 1}-\frac{1}{\rho_{f}} \partial_{i} \widetilde{p}^{*}-\partial_{j} \widetilde{\tau}_{i j}+g\left(\frac{\widetilde{\theta}_{v}-\left\langle\widetilde{\theta}_{v}\right\rangle}{\left\langle\widetilde{\theta}_{v}\right\rangle}\right) \delta_{i, 3}+\frac{1}{\rho_{f}} S_{i}^{m}, \\
\partial_{i} \widetilde{u}_{i} & =0 \\
\partial_{t} \widetilde{\theta}+\widetilde{u}_{j} \partial_{j} \widetilde{\theta} & =-\partial_{j} \widetilde{\pi}_{j}+\frac{1}{\rho_{f} c_{p, f}}\left(S^{h}\right), \\
\partial_{t} \widetilde{q}+\widetilde{u}_{j} \partial_{j} \widetilde{q} & =-\partial_{j} \widetilde{\Omega}_{j}+S^{q}, \\
\widetilde{\theta}_{v} & =\widetilde{\theta}(1+0.61 \widetilde{q}) .
\end{aligned}
$$

In the above equations, $u_{i}$ are the three velocity components, $\theta$ and $\theta_{v}$ are the potential and virtual potential temperature respectively and $q$ is the specific humidity. The overhead tildes $(\sim)$ represent quantities filtered via spectral cutoff while the brackets $(\langle\rangle)$ represent horizontal averaging. Note that in this study, since we simulating the atmospheric surface layer, 
temperature and potential temperature are considered to be the same. The modified pressure term $\widetilde{p}^{*}$ consists of static pressure $(p)$, filtered kinetic energy $\left(0.5 \widetilde{u}_{i} \widetilde{u}_{i}\right)$ and the trace of the subgrid-scale tensor $\left(\tau_{k k} / 3\right)$. The first term on the R.H.S of Eq. (S1) is the large-scale pressure gradient that is imposed to drive the simulation. Terms $\widetilde{\tau}, \widetilde{\pi}, \widetilde{\Omega}$ represent the subgrid-scale (SGS) fluxes for momentum, heat and specific humidity respectively. The source/sink terms $S_{i}^{m}, S^{h}$ and $S^{q}$ are included to incorporate the

5 effect of sublimating particles being transported by the wind on the flow dynamics and thermodynamics. Particles exert drag on the flow and exchange heat and mass due to sublimation/deposition. In accordance with the incompressible assumption, we consider the density of the fluid $\rho_{f}$ to remain constant. The specific heat capacity of air $c_{p . f}$ is considered to be constant irrespective of the value of specific humidity.

\section{S1.2 SGS model}

10 The deviatoric part of the SGS flux tensor for momentum is computed using a dynamic Smagorinsky-type model, while the scalar SGS fluxes are related to the momentum fluxes using fixed turbulent Prandtl $(P r)$ and Schmidt $(S c)$ numbers. The equations used for SGS fluxes are

$\tau_{i j}=-2 \nu_{T} \widetilde{S_{i j}}=-2 c_{s}^{2} \Delta^{2}|\widetilde{S}| \widetilde{S_{i j}}$,

$\pi_{j}=-\frac{\nu_{T}}{\operatorname{Pr}} \partial_{j} \tilde{\theta}$,

15

$\Omega_{j}=-\frac{\nu_{T}}{S c} \partial_{j} \widetilde{q}$,

where, $\nu_{t}$ is the turbulent viscosity of air which is parametrized using a Smagorinsky-type eddy-viscosity approach. In this approach, $\nu_{T}$ is considered to be equal to the product of a length scale $c_{s} \Delta$ times a velocity scale $c_{s} \Delta\left|\widetilde{S_{i j}}\right| . \widetilde{S_{i j}}$ is symmetric part of the strain rate tensor and $\left|\widetilde{S_{i j}}\right|=\sqrt{2 \widetilde{S_{i j} \widetilde{S_{i j}}}}$ is the Galilean invariant estimate of $\widetilde{S_{i j}}$. The Smagorinsky coefficient, $c_{s}$ is estimated dynamically using resolved velocity values through applying the Germano identity along trajectories of lagrangian fluid particles. This approach, known as the Lagrangian Scale-Dependent Dynamic Smagorinsky (LASD) SGS model was developed by Bou-Zeid et al. (2005), where detailed derivations and applications for LES can be found.

\section{S1.3 Lower-boundary condition for Eulerian quantities}

For the surface boundary conditions for velocity, a non-slip condition is imposed for the vertical velocity and for the horizontal components of the momentum equation an equivalent shear stress is imposed using the log-law with correction for the presence of particles between the surface and the first grid point based on Shao and Li (1999),

$\tau_{i, 3}\left(x, y, z_{1}\right)=\left[\frac{\sqrt{\left(\widehat{\widetilde{u}}_{1}^{2}+\widehat{\widetilde{u}}_{2}^{2}\right)}}{\ln \left(z_{1} / z_{0}\right)}\right]^{2} \mathbf{n}_{\mathbf{i}}+\frac{1}{\rho_{f}} S_{i}^{m}\left(x, y, z_{1}\right) z_{1}$,

where $i$ represents the direction in the plane parallel to the surface $(i=1,2)$, and $\mathbf{n}_{\mathbf{i}}$ is a unitary directional vector defined as: $\mathbf{n}_{\mathbf{i}}=\widehat{\widetilde{u}}_{i} / \sqrt{\widehat{\widetilde{u}}_{1}^{2}+\widehat{\widetilde{u}}_{2}^{2}}$. The shear stress is imposed at $z_{1}=\Delta z / 2$. Recall that $\left(1 / \rho_{f}\right) S_{i}^{m}$ is the sink of momentum due to drag forces from the flow-particle interaction. In the case with no particles, the stress term reconciles with the standard log law 
whereas in the presence of the particles, the stress at the surface decreases $\left(S_{i}^{m}\right.$ is negative). The $(\widehat{\approx})$ operator represents a double filtering operation (at $2 \Delta$ grid spacing) on horizontal components of the flow, which is equivalent to a local average. The surface roughness is constant over the entire numerical domain and has a value of $z_{0}=10^{-5} \mathrm{~m}$. For the numerical integration of the momentum equation, the vertical derivatives of the horizontal velocity components are also needed. These are parametrised

5 at the first grid point also using Monin-Obukhov similarity theory,

$\partial_{3} \widetilde{u}_{i}\left(x, y, z_{1}\right)=\left(\frac{\sqrt{\tau_{i, 3}}}{\kappa z_{1}}\right) \mathbf{n}_{\mathbf{i}}$,

with $\tau=\sqrt{\tau_{1,3}^{2}+\tau_{2,3}^{2}}$ and $\kappa$ is the von Karman constant $=0.4$. The kinematic sensible $\left(\overline{w^{\prime} \theta^{\prime}}\right)$ and latent $\left(\overline{w^{\prime} q^{\prime}}\right)$ heat fluxes are zero.

\section{S1.4 Surface-flow coupling}

10 Ice grains lying on the surface enter into the overlying, usually turbulent fluid through mechanisms of aerodynamic, rebound and splash entrainment from the surface. These mechanisms and the accompanying models used in this work are described in detail in the following paragraphs. There are some common elements in these models. All particles are assumed to enter the flow at a height of $h_{\text {init }}=4\left\langle d_{p}\right\rangle$ above the surface. Each of these models provides the number of particles per unit area that enter into the flow, along with the initial conditions for both velocity magnitude and direction for the lagrangian particle.

\section{S1.4.1 Aerodynamic entrainment}

Initiation of aeolian transport of particles occurs due to entrainment of particles by the fluid when the stress at the surface $\tau_{f, \text { surface }}$ increases above a threshold value $\left(\tau_{\text {threshold }}\right)$ and particles from the surface are dislodged to relieve stress at the surface. Bagnold (1941) derived an expression for the threshold stress as

$\tau_{\text {threshold }}=A^{2} g\left\langle d_{p}\right\rangle\left(\rho_{p}-\rho_{f}\right)$

20 where $\mathrm{g}$ is the acceleration due to gravity, $\left\langle d_{p}\right\rangle$ is the mean particle diameter of all particles present on the surface, $\rho_{p}$ and $\rho_{f}$ are the density of the particles and the fluid respectively. The coefficient $A$ is an empirical parameter taken to be equal to 0.2 based on wind tunnel experiments of drifting and blowing snow by Clifton et al. (2006).

From each computational grid point on the surface, the number of particles entering the fluid due to aerodynamic entrainment at each time-step can then be computed using the expression of Anderson and Haff (1991) as

$N_{a e}=\frac{C_{e}}{8 \pi\left\langle d_{p}\right\rangle^{2}}\left(\tau_{f, \text { surface }}-\tau_{\text {threshold }}\right) \Delta x \Delta y \Delta t$,

where $C_{e}$ is an empirically derived constant of proportionality ( equal to 1.5, according to Doorschot and Lehning (2002); Groot Zwaaftink et al. (2014) and $\Delta x$ and $\Delta y$ are the grid step sizes in the streamwise and cross-stream directions respectively and $\Delta t$ is the simulation time-step. Once the number of particles has been calculated, for each particle entrained into the flow, the diameter is sampled from the log-normal distribution of the particle size distribution (characterized by the $\left\langle d_{p}\right\rangle$ and $\sigma_{d_{p}}$ ). 
The particle is initialized at height $h_{\text {init }}$ and provided with an initial velocity magnitude sampled from a log-normal distribution characterized by mean velocity of $3.5 u_{*}$ and a standard deviation of $2.5 u_{*}$. The ejection angle is sampled from a lognormal distribution with a mean angle as a function of mean particle diameter as follows,

$\left\langle\alpha_{\text {ae,ejection }}\right\rangle=75-55\left(1-\exp \frac{-\left\langle d_{p}\right\rangle}{175 \times 10^{-6}}\right)$.

5 Thus, at any given time-step, based on the stress at the wall, the number of particles entering the fluid along with the initial position and velocity can be simulated.

\section{S1.4.2 Rebound and splash entrainment}

A particle, once entrained within the turbulent fluid, may impact the bed upon which it may rebound back into the fluid (known as rebound entrainment) as well as dislodge particles present in the bed that get entrained into the fluid as well (known as splash entrainment).

Consider a particle with diameter $d_{i}$ that impacts the bed with velocity magnitude $v_{i}$ at a verticle impact angle of $\alpha_{i}$. The probability that the particle shall rebound following impact $\left(P_{r}\right)$ is given by Anderson and Haff (1991)

$$
P_{r}=P_{m}\left(1-e^{-\gamma v_{i}}\right)
$$

where $P_{m}$ is the maximum probability that is assumed to be equal to 0.9 for snow (Groot Zwaaftink et al., 2011) and $\gamma$ is a empirical coefficient equal to 2 according to Anderson and Haff (1991). Once the particle rebounds, its velocity must be re-initialized. The rebound velocity magnitude $v_{r}$ is taken to be half of $v_{i}$. The vertical rebound angle $\left(\alpha_{r}\right)$ is sampled from an exponential distribution of mean angle equal to $45^{\circ}$ following (Kok and Renno, 2009). The particle is assumed to remain in the same vertical plane before and after the collision for simplicity.

As mentioned earlier, the impacting particle may break inter-particle bonds present in the snowbed and dislodge additional additional particles into the overlying turbulent flow. The number of particles dislodged $N_{\text {splash }}=\min \left(N_{E}, N_{M}\right)$, where,

$$
\begin{gathered}
N_{E}=\frac{\left(1-P_{r} \epsilon_{r}-\epsilon_{f}\right) d_{i}^{3} v_{i}^{2}}{2\langle v\rangle^{2}\left(\langle d\rangle+\frac{\sigma_{d}^{2}}{\langle d\rangle}\right)^{3}\left(1+r_{E} \sqrt{5\left[1+\left(\frac{\sigma_{d}}{\langle d\rangle}\right)^{2}\right]^{9}-5}\right)+2 \phi}, \\
N_{M}=\frac{\left(1-P_{r} \mu_{r}-\mu_{f}\right) d_{i}^{3} v_{i} \cos \alpha_{i}}{\langle v\rangle\left(\langle d\rangle+\frac{\sigma_{d}^{2}}{\langle d\rangle}\right)^{3}\left(\langle\cos \alpha\rangle\langle\cos \beta\rangle+r_{M} \sqrt{\left[1+\left(\frac{\sigma_{d}}{\langle d\rangle}\right)^{2}\right]^{9}-1}\right)}
\end{gathered}
$$

In the above equations, $P_{r}$ is the probability of rebound of the impacting grain, calculated using Eq. (S14); $\epsilon_{f}$ and $\epsilon_{r}$ are the fractions of impact energy lost to the bed due to friction and that retained by the rebounding particle respectively. The corresponding fractions of impact momentum are denoted as $\mu_{f}$ and $\mu_{r}$ respectively. The mean and standard deviation of the ejecta's diameter are represented by $\langle d\rangle$ and $\sigma_{d}$. $\langle v\rangle$ is the mean velocity of the ejecta with $\alpha$ and $\beta$ being the vertical and 
horizontal ejection angles of ejected particles. Cohesion of the snow bed is represented by $\phi$. Correlation coefficient between the ejected mass $m$ and velocity $v^{2}$ is $r_{E}$ while $r_{M}$ is the corresponding correlation coefficient between $m$ and $v$.

The splashed particles are initialized at a height similar to the aerodynamically entrained particles at the height of $h_{\text {init }}$. The initial velocity magnitude is assumed to follow an exponential distribution with a mean value of $0.25 v_{i}^{0.3}$. The vertical

5 entrainment angle $\alpha$ is chosen as a random number that is drawn from an exponential distribution with a mean value of $50^{\circ}$. The horizontal splash angle $\beta_{n}$ is sampled from a Gaussian distribution with a mean value of the horizontal angle of the impacting particle and a standard deviation of $15^{\circ}$.

Models for splash entrainment are dervied by Comola and Lehning (2017) and similar models have previously been implemented by Groot Zwaaftink et al. (2014). Additional references can be found in these works.

\section{S1.5 Dynamics of particles in flow}

Once the ice grain is entrained within the turbulent flow, its fate is determined by solving the equations of motion. The only forces we included in our calculations are drag forces and gravity. The drag forces are computed by considering the ice grains to be spherical and use well-known bulk parametrizations of drag on a sphere in a turbulent flow (Clift et al., 1978). The equations for motion are as follows

$$
\begin{aligned}
\frac{d u_{p, i}}{d t} & =\left(1+0.15 R e_{p}^{0.687}\right) \frac{1}{t_{p}}\left(\widetilde{u}_{i}+u_{s g s, i}-u_{p, i}\right)-g \delta_{i, 3} \\
t_{p} & =\rho_{p} d_{p}^{2} / 18 \rho_{f} \nu_{a}
\end{aligned}
$$

where, $u_{p, i}$ are the velocity components of the particle, $\widetilde{u}_{i}$ is the filtered velocity solved in Eq. (S1), $R e_{p}$ is the particle Reynolds number (defined later), $\nu_{a}$ is the kinematic viscosity of air and $u_{s g s, i}$ is the SGS fluid velocity computed along the path of the particle's motion. We use the equation for SGS velocities from Weil et al. (2004) as follow,

$\mathrm{d} u_{s g s, i}=-f \frac{u_{s g s, i}}{T_{f}} \mathrm{~d} t+\frac{1}{2}\left(\frac{1}{\sigma^{2}} \frac{\mathrm{d} \sigma^{2}}{\mathrm{~d} t} u_{s g s, i}+\frac{\partial \sigma^{2}}{\partial x_{i}}\right) \mathrm{d} t+\left(f \frac{2 \sigma^{2}}{T_{f}}\right)^{1 / 2} \mathrm{~d} \xi_{i}$

where $\sigma^{2}=2 e / 3$ is the variance of the SGS velocity, which is directly proportional to the SGS turbulence kinetic energy (TKE) e. The fraction of TKE present in subgrid scales is represented by f. $\mathrm{d} \xi_{i}$ is a normally distributed random number with zero mean and a variance of $\mathrm{d} t$. The velocity autocorrelation timescale $\left(T_{f}=2 \sigma^{2} / C_{o} e\right)$ is used to consider the effect of heavy particles as opposed to purely lagrangian particles without inertia.

The mass exchange between the ice grain and the surrounding fluid is driven by the difference between the vapour density at the surface of the particle (which is considered to be saturated) and the vapour density in the fluid at the particle location. We assume that the total density of the fluid $\rho_{f}$ is constant and thus can directly write the mass balance equation as a function 
of specific humidity as follows

$$
\begin{aligned}
\frac{\mathrm{d} m_{p}}{\mathrm{~d} t} & =\pi \mathscr{D} d_{p} \rho_{f}\left(\widetilde{q}-q_{p, \text { surface }}\right) \mathcal{S} h \\
q_{p, \text { surface }} & =\frac{M_{W}}{R_{u} T_{p} \rho_{f}} e_{s, a} \exp \left[\frac{L_{s} M_{w}}{R_{u}}\left(\frac{1}{T_{f}}-\frac{1}{T_{p}}\right)\right], \\
e_{s, a}\left(T_{f}\right) & =611.15 \exp \left[\left(23.036-\frac{T_{f}-273.15}{333.7}\right)\left(\frac{T_{f}-273.15}{6.67+T_{f}}\right)\right] .
\end{aligned}
$$

5 In above equation, $m_{p}$ is the mass of the particle, $\mathscr{D}$ is the diffusion coefficient of vapour in air, $\widetilde{q}$ is the filtered specific humidity computed using Eq. (S4), $\mathcal{S} h$ is the Sherwoord number (described later) and $q_{p, \text { surface }}$ is the specific humidity at the surface, which is computed using the ideal gas law and the Clasius-Clayperon equation, integrated between the particle surface and the mass-boundary layer. $M_{W}$ is the molecular weight of water, $R_{u}$ is the universal gas constant and $L_{s}$ is the latent heat of sublimation. The saturation vapour pressure with respect to ice, $e_{s, a}$ is computed using the Arden-Buck equation for ice.

The temperature of the particle evolves according to a balance between the latent heat due to mass exchange and the convective heat transfer between the ice grain and the surrounding fluid and can be expressed as,

$c_{i} m_{p} \frac{\mathrm{d} T_{p}}{\mathrm{~d} t}=L_{s} \frac{\mathrm{d} m_{p}}{\mathrm{~d} t}+\pi \mathscr{K} d_{p}\left(\widetilde{\theta}-T_{p}\right) \mathcal{N} u$,

where $c_{i}$ is the specific heat capcity of ice, $\mathscr{K}$ is the thermal conductivity of the air, $\widetilde{\theta}$ is the filtered potential temperature obtained by solving Eq. (S3) and $\mathcal{N} u$ is the Nusselt number.

The effect of turbulence on momentum, heat and mass transfer is neatly encompassed in three non-dimensional parameters, namely the Reynolds, Nusselt and Sherwood numbers respectively. We use the following expressions to calculate these numbers.

$$
\begin{aligned}
R e_{p} & =\frac{d\left|\boldsymbol{u}_{\text {rel }}\right|}{\nu_{\text {air }}}, \\
\mathcal{N} u & =1.79+0.606 \operatorname{Re}_{p}^{1 / 2} \operatorname{Pr}^{1 / 3}, \\
20 \quad \mathcal{S} h & =1.79+0.606 \operatorname{Re}_{p}^{1 / 2} S c^{1 / 3},
\end{aligned}
$$

The coupling between the particles and the flow occurs through the source/sink terms in equations for momentum (Eq. (S1), $S_{m}$ ), temperature (Eq. (S3), $S^{h}$ ) and specific humidity (Eq. (S4), $S^{q}$ ). Consider a particle lying at a point within in the fluid. The eight closest points of the LES discretized grid shall necessarily form a cube considering that we use a structured Cartesian grid. The point force due to drag upon the particle is simply extrapolated onto these eight points using inverse distance weighting. The total force at any LES grid point is a summation of all such extrapolated forces. If an LES grid point has $N_{p}$ particles 
contributing to the total force at it's location, the source/sink terms can be simply represented as

$$
\begin{aligned}
S_{i}^{m} & =-\sum_{\beta=1}^{N_{p}} \frac{w_{\beta}}{\Delta V}\left(m_{p} \frac{\mathrm{d} u_{p . i}}{\mathrm{~d} t}+\frac{\mathrm{d} m_{p}}{\mathrm{~d} t} u_{p . i}\right)_{\beta}, \\
S^{h} & =-\sum_{\beta=1}^{N_{p}} \frac{w_{\beta}}{\Delta V}\left(\pi \mathscr{K} d_{p}\left(\widetilde{\theta}-T_{p}\right) \mathcal{N} u\right)_{\beta}, \\
S^{q} & =-\sum_{\beta=1}^{N_{p}} \frac{w_{\beta}}{\Delta V} \frac{1}{\rho_{f}}\left(\frac{\mathrm{d} m_{p}}{\mathrm{~d} t}\right)_{\beta} .
\end{aligned}
$$

5 In the above equation, $w_{\beta}$ is the weight for the extrapolation operation computed using inverse distance weighting.

\section{S1.6 Numerics}

The flow equations ( Eq.(S1), (S3) and (S4) ) are solved in the Eulerian frame of reference using a pseudo-spectral collocation method in the same fashion as that introduced by Moeng (1984) and Albertson and Parlange (1999). In this approach, the horizontal gradient operations are computed using fourier methods while vertical gradients are computed using second order finite differences. Because of the fourier based methods in both horizontal directions, the domain is fully periodic and no lateral boundary conditions are needed, and hence, the domain size tends to infinity in practical effects. The use of fourier methods is quite useful for SGS modelling is because allows the direct use of spectral cut-off for filtering the flow quantities. The horizontal grid is uniform with equal discretizations in the both the streamwise and cross-stream directions. In the vertical direction we use hyperbolic tangent function based grid stretching. This to insure that there are sufficient grid points within the saltation region. Incompressibility is enforced through a prediction-correction approach where the velocity is marched in time and the poisson equation is used to solve for the dynamic pressure field. To maintain proper consistency in numerics for solving the poisson equation, the vertical grid is staggered to ensure second-order accuracy.

Integration in time is done using a second-order Adams-Bashforth scheme. In addition, the non-linear convective terms are dealaised with the $3 / 2$ rule (Canuto et al., 1988). The code is fully parallelised using Message Passing Interface (MPI), and the FFTW library (Frigo and Johnson, 2005) is employed for the Fourier transforms. The pressure solver is based on the pipeline Thomas algorithm (Povitsky and Morris, 2000) and is used to enforce incompressibility implied by Eq. (S2).

The equation of motion for particles (Eq. (S17)) along with equations for heat and mass budgets (Eq. (S20) and (S21)) are solved using a simple first-order time stepping using forward euler method. At each timestep, information from the Eulerian LES grid is translated to locations of the lagrangian particles using simple linear interpolation. The source/sink terms due to the flow-particle coupling are translated back to the Eulerian frame of reference using linear extrapolation as described in Eq. (S25).

All simulations in this study are driven by an imposed large-scale pressure gradient $\mathscr{P}=-\left(1 / \rho_{f}\right) \partial \widetilde{p_{\infty}} / \partial x$ in the streamwise direction. This quantity is kept constant throughout the simulation. Upon choosing values of the surface friction velocity $u_{*}$, the imposed pressure gradient can be computed as $\mathscr{P}=u_{*}^{2} / L_{z}$, where $L_{z}$ is the vertical extent of the computational domain. 


\section{S2 Physical constants and numerical parameters used in the study}

Table S1. Important Physical Parameters and Constants

\begin{tabular}{llc}
\hline Parameter & Symbol [Units $]$ & Value \\
\hline Latent heat of sublimation & $L_{s}\left[\mathrm{~J} \mathrm{~kg}^{-1}\right]$ & $2835.49 \times 10^{3}$ \\
Prandtl number & $\operatorname{Pr}[-]$ & 0.72 \\
Schmidt number & $S c[-]$ & 0.63 \\
Molecular Weight of Water & $M_{w}\left[\mathrm{~kg} \mathrm{~mol}^{-1}\right]$ & 0.018015 \\
Density of ice & $\rho_{p}\left[\mathrm{~kg} \mathrm{~m}^{-3}\right]$ & 918.4 \\
Density of air & $\rho_{f}\left[\mathrm{~kg} \mathrm{~m}^{-3}\right]$ & 1.34 \\
Specific heat capacity of air & $c_{p, f}\left[\mathrm{~J} \mathrm{~kg}^{-1} \mathrm{~K}^{-1}\right]$ & $1.005 \times 10^{3}$ \\
Specific heat capacity of ice & $c_{i}\left[\mathrm{~J} \mathrm{~kg}^{-1} \mathrm{~K}^{-1}\right]$ & $2.0357 \times 10^{3}$ \\
Roughness length & $z_{0}\left[\mathrm{~m}^{-1}\right.$ & $1.0 \times 10^{-5}$ \\
Cohesion Strength & $\phi[\mathrm{J}]$ & $10^{-10}$ \\
Diffusivity of vapor in air & $\mathscr{D}\left[\mathrm{m}^{2} \mathrm{~s}^{-1}\right]$ & $1.96 \times 10^{-5}$ \\
Thermal conductivity of air & $\mathscr{K}\left[\mathrm{W} \mathrm{m}^{-1} \mathrm{~K}^{-1}\right]$ & 0.023 \\
Universal Gas Constant & $R_{u}\left[\mathrm{~J} \mathrm{~mol}^{-1} \mathrm{~K}^{-1}\right]$ & 8.314 \\
Kinematic Viscosity of air & $\nu_{a}\left[\mathrm{~m}^{2} \mathrm{~s}^{-1}\right]$ & $1.24 \times 10^{-5}$ \\
& & \\
\hline
\end{tabular}

Table S2. Details of the numerical setup

\begin{tabular}{llc}
\hline Parameter & Symbol [Units] & Value \\
\hline Streamwise extent of domain & $L_{x}[\mathrm{~m}]$ & 6.4 \\
Cross-stream extent of domain & $L_{y}[\mathrm{~m}]$ & 6.4 \\
Vertical extent of domain & $L_{z}[\mathrm{~m}]$ & 6.4 \\
Grid points in horizontal directions & $\{n x, n y\}[-]$ & 64 \\
Grid points in vertical direction & $n z[-]$ & 128 \\
Vertical grid stretching & $\left\{z_{\min }, z_{\max }\right\}[\mathrm{m}]$ & $\{0.01,0.1\}$ \\
Time step & $\Delta t[\mu s]$ & 50 \\
Total time & $T[s]$ & 1200 \\
& & \\
\hline
\end{tabular}




\section{S3 Supplementary results}

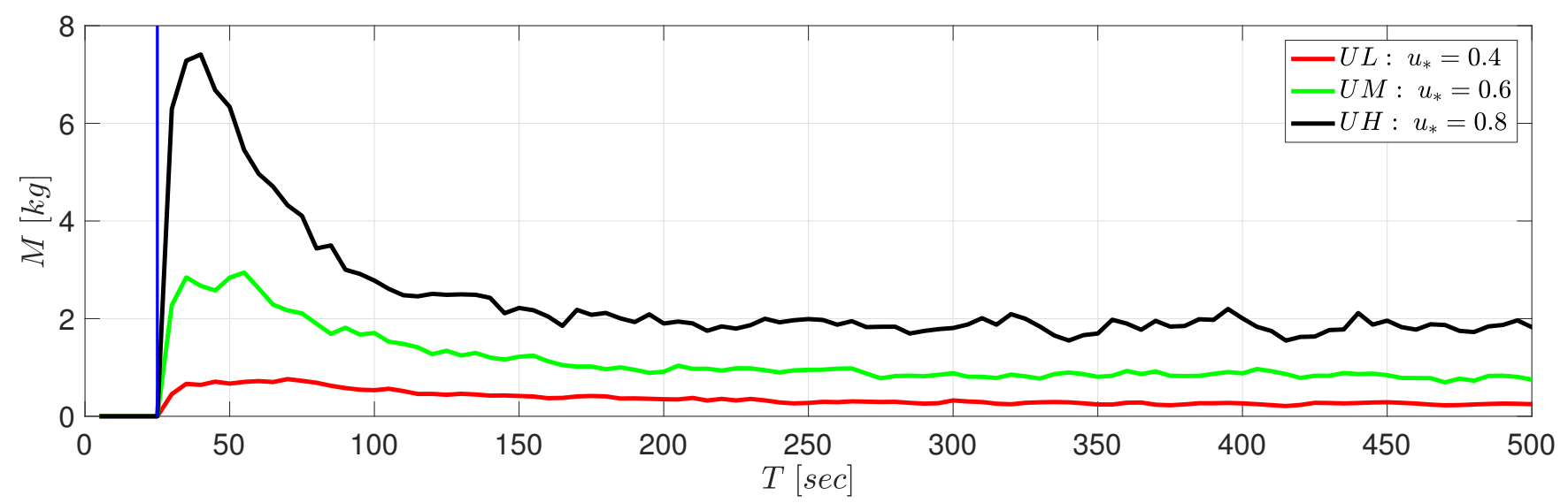

Figure S1. The total mass of snow presented in the fluid at any given time of the simulation for three different forcing conditions. The vertical blue line denotes the commencement of surface erosion.

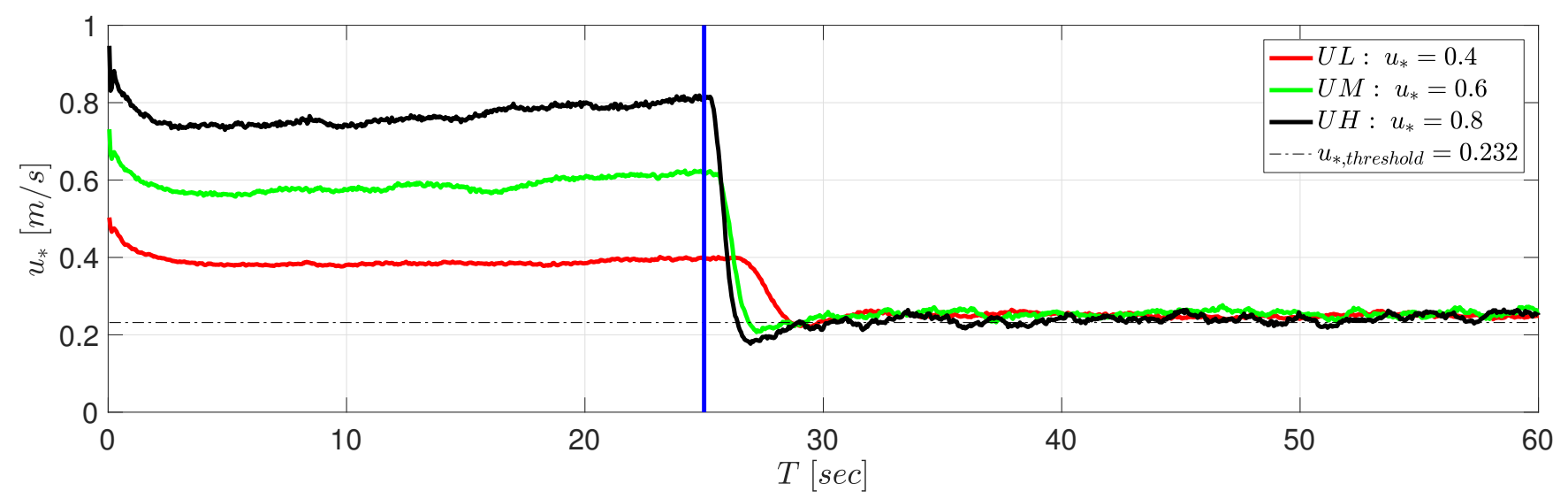

Figure S2. Evolution of the friction velocity at the surface $\left(u_{*}\right)$. The vertical blue line denotes the commencement of surface erosion which until this point in time has been artificially restricted.

We present here two results that supplement the results presented in the main portion of the letter. While these results are not critical to discussions presented in the letter, they are nevertheless interesting in their own right and help justify certain assumptions used in the simulation setup.

Fig. S1 shows evolution of the total air-borne mass of snow under three different forcing conditions. It shows that that once surface erosion is allowed to occur, there is a rapid increase in the total mass present in the atmosphere, following which, it slowly decays to reach its asymptotic value at approximately 300 seconds. All the three forcings, marked by the three initial $u_{*}$ values of $0.4(\mathrm{UL}), 0.6(\mathrm{UM})$ and $0.8(\mathrm{UH})$ show similar behaviour with larger mass entrained by the air with increasing $u_{*}$ 
values as expected. The results in Fig. S1 explain the increase in errors in the mass-loss rate with increasing $u_{*}$ values between the Nu-TM and TM approaches as discussed in Fig. 3 of the letter.

In Fig. S2, the evolution of the surface friction velocity is shown as a function of time. The vertical blue line marks the beginning of surface erosion. It shows that irrespective of the forcing used to drive the flow, once saltation begins, the friction

5 velocity at the surface drops to that of $u_{*, \text { threshold }}$. The value of $u_{*, \text { threshold }}$, computed using Eq.(S11) for $\tau_{\text {threshold }}$ and by considering that $u_{*, \text { threshold }}=\sqrt{\tau_{\text {threshold }} / \rho_{f}}$ is found to be equal to 0.232 . The rate at which the surface friction velocity decays is dependent on the forcing used with larger forcing decaying more rapidly to the asymptotic value of $u_{*, \text { threshold }}$. This result is important since the surface friction velocity dictates the turbulent exchange between the surface and the overlying turbulent fluid. A drop in the surface friction velocity shows during drifting and blowing snow events, the heat and mass transfer 10 between the surface and the atmosphere can be neglected as a first-order approximation and can be modelled using fixed stress values in more detailed studies. 


\section{References}

Albertson, J. and Parlange, M.: Natural integration of scalar fluxes from complex terrain, Water Resour. Res., 23, 239-252, 1999.

Anderson, R. and Haff, P.: Wind modification and bed response during saltation of sand in air, in: Aeolian Grain Transport 1, pp. 21-51, Springer, 1991.

5 Bagnold, R. A.: The physics of blown sand and desert dunes, New York: Methuen, 1941.

Bou-Zeid, E., Meneveau, C., and Parlange, M.: A scale-dependent Lagrangian dynamic model for large eddy simulation of complex turbulent flows, Physics of Fluids, 17, 025 105, https://doi.org/10.1063/1.1839152, http://dx.doi.org/10.1063/1.1839152, 2005.

Canuto, C., Hussaini, M., Quarteroni, A., and Zang, T.: Spectral Methods in Fluid Dynamics, Springer-Verlag, Berlin, 1988.

Clift, R., Grace, J., Weber, M., and Bubbles, D.: Particles, Academic, New York, pp. 171-202, 1978.

Clifton, A., Rüedi, J.-D., and Lehning, M.: Snow saltation threshold measurements in a drifting-snow wind tunnel, Journal of Glaciology, 52, 585-596, 2006.

Comola, F. and Lehning, M.: Energy- and momentum-conserving model of splash entrainment in sand and snow saltation, Geophysical Research Letters, 44, 1601-1609, https://doi.org/10.1002/2016GL071822, http://dx.doi.org/10.1002/2016GL071822, 2016GL071822, 2017.

Doorschot, J. J. J. and Lehning, M.: Equilibrium Saltation: Mass Fluxes, Aerodynamic Entrainment, and Dependence on Grain Properties, Boundary-Layer Meteorology, 104, 111-130, https://doi.org/10.1023/A:1015516420286, https://doi.org/10.1023/A:1015516420286, 2002.

Frigo, M. and Johnson, S.: The design and implementation of FFTW3, Proceedings of the IEEE, 93, 216-231, 2005.

Groot Zwaaftink, C. D., Löwe, H., Mott, R., Bavay, M., and Lehning, M.: Drifting snow sublimation: A high-resolution 3-D model with temperature and moisture feedbacks, Journal of Geophysical Research: Atmospheres, 116, n/a-n/a, https://doi.org/10.1029/2011JD015754, http://dx.doi.org/10.1029/2011JD015754, d16107, 2011.

Groot Zwaaftink, C. D., Diebold, M., Horender, S., Overney, J., Lieberherr, G., Parlange, M. B., and Lehning, M.: Modelling Small-Scale Drifting Snow with a Lagrangian Stochastic Model Based on Large-Eddy Simulations, Boundary-Layer Meteorology, 153, 117-139, https://doi.org/10.1007/s10546-014-9934-2, https://doi.org/10.1007/s10546-014-9934-2, 2014.

Kok, J. F. and Renno, N. O.: A comprehensive numerical model of steady state saltation (COMSALT), Journal of Geophysical Research: Atmospheres, 114, n/a-n/a, https://doi.org/10.1029/2009JD011702, http://dx.doi.org/10.1029/2009JD011702, d17204, 2009.

Moeng, C. H.: A large-eddy simulation model for the study of planetary boundary-layer turbulence., J. Amtos. Sci., $41,1984$.

Povitsky, A. and Morris, P. J.: A higher-order compact method in space and time based on parallel implementation of the Thomas algorithm, J. Comput. Phys., 161, 182-203, 2000.

Shao, Y. and Li, A.: Numerical Modelling of Saltation in the Atmospheric Surface Layer, Boundary-Layer Meteorology, 91, 199-225, https://doi.org/10.1023/A:1001816013475, https://doi.org/10.1023/A:1001816013475, 1999.

Weil, J. C., Sullivan, P. P., and Moeng, C.-H.: The Use of Large-Eddy Simulations in Lagrangian Particle Dispersion Models, Journal of the Atmospheric Sciences, 61, 2877-2887, https://doi.org/10.1175/JAS-3302.1, https://doi.org/10.1175/JAS-3302.1, 2004. 